\title{
Quantum States of Matter of Simple Bosonic Systems: BEC's, Superfluids and Quantum Solids
}

\author{
Zaira Nazario ${ }^{\dagger}$ and David I. Santiago ${ }^{\dagger, \star}$ \\ $\dagger$ Department of Physics, Stanford University,Stanford, California 94305 \\ * Gravity Probe B Relativity Mission, Stanford, California 94305
}

(Dated: November 11, 2018)

\begin{abstract}
The phase diagram of a single component Bose system in a lattice at zero temperature is obtained. We calculate the variational energies for the Mott insulating and superfluid phases. Below a certain critical density the Mott insulating phase is stable over the superfluid phase for low enough tunelling amplitude regardless of whether the number of bosons is or is not incommensurate with the lattice. The transition is discontinuous as the superfluid order parameter jumps from a finite value to zero at the Mott transition.
\end{abstract}

PACS numbers: 03.75.Hh, 03.75.Nt, 068.18.Jk, 5.30.Jp

The purpose of the present work is to study the phase diagram of a single component Bose gas at zero temperature. Specifically, we have in mind a system of bosons in an optical lattice, where the wells on each site are made deeper thus causing a transition from the superfluid phase into a phase where bosons are localized on the lattice sites, i.e a quantum solid or Mott insulating phase ${ }^{1.2}$. Recently such a transition has been observed experimentally ${ }^{1}$.

The original theoretical work by M.P.A. Fisher et. $a l^{2}{ }^{2}$ on the superfluid-Mott transition in bosonic systems predated the experimental realization of artificially engineered Bose-Einstein condensates ${ }^{3.4 .5}$ (BEC's), which are superfluid systems as they posses a finite sound speed ${ }^{6.11 .14}$ and suppressed long-wavelength scattering 7.8 . In that original work the transition was asserted to be continuous and possible only for a number of bosons commensurate with the lattice, i.e. integral number of bosons per site. While widely believed to be true, both assertions are quite puzzling and may contradict experiment ${ }^{15}$. In fact, recent theoretical ${ }^{19}$ and numerical work ${ }^{10}$ has pointed to a transition that is incommensurate and "nonstandard" even for a pure system $^{10}$. In the present note we extend the work in the pure system within the Bogolyubov approximation for the superfluid 11.12

The superfluid-Mott transition is between a superfluid and a quantum solid. In systems without disorder, fluidsolid transitions are usually discontinuous as the order parameters are too different 17 . In the superfluid system that is certainly the case. The Mott insulating phase is characterized by a well defined number of bosons per site, one could take the density as its order parameter. The superfluid phase has off-diagonal order with breaking of gauge invariance $\frac{18}{}$, i.e. $U(1)$, characterized by a coherent ground state of Bogolyubov pairs 11 . The transition will be characterized by a discontinuous jump in the superfluid response ${ }^{15}$.

We calculate the variational energies of Mott insulating and superfluid phases with both commensurate and incommensurate number of bosons. We find that below some critical value $r_{c}$ of the ratio $t / U$ the Mott insulating phase is energetically favorable over the superfluid phase as long as the number of bosons per site is not too high, i.e. less than a critical value $n_{c}$. Below $r_{c}$ and $n_{c}$ the superfluid phase is never stable regardless of commensurability.

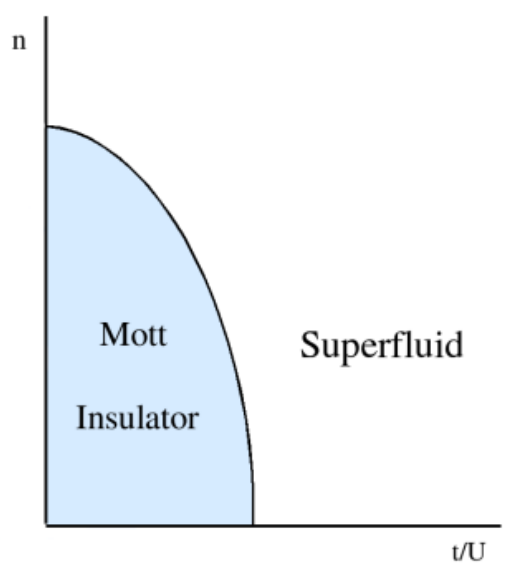

FIG. 1: Schematic Phase Diagram of a single component Bose system at zero temperature. $\mathrm{n}$ is the average number of bosons per site.

When the number of bosons per site is higher than the critical number $n_{c}$, the quantum solid phase is never stable within our approximations and there is only a superfluid phase even for arbitrarily small $t / U$. On the other hand, we believe our approximations, which are apt at low number of bosons per site, fail at high densities and there will be "crossover" to physics that is more like that of Josephson coupled arrays 2,16 . This is a matter for further study. The phase diagram is shown in figure 1 .

A very apt prototype Hamiltonian to study bosons in a lattice (which, of course can be an optical lattice) is the Bose Hubbard Hamiltonian: 


$$
\begin{array}{r}
\mathcal{H}=\frac{-t}{2} \sum_{<i j>}\left(a_{i}^{\dagger} a_{j}+a_{j}^{\dagger} a_{i}\right)+U \sum_{i} a_{i}^{\dagger} a_{i}\left(a_{i}^{\dagger} a_{i}-1\right) \\
-\varepsilon \sum_{i} a_{i}^{\dagger} a_{i}
\end{array}
$$

where $t$ is the nearest-neighbor tunneling or hopping amplitude, $U$ is the on-site repulsion, and $\varepsilon$ is the well depth for each lattice site. We will suppose our lattice to have $N$ sites and our system to have $M$ bosons with $M / N$ no necessarily an integer.

When $U=0$, the ground state of such a Hamiltonian is a BEC with all atoms in the zero momentum state. Such a system is not superfluid as its excitation spectrum has quadratic dispersion ${ }^{14}$. An arbitrary small amount of repulsion causes the system to order and become superfluid. The ground state, first found by Bogolyubov ${ }^{11}$, is

$$
\left|\psi_{0}\right\rangle=\prod_{\mathbf{k}} \frac{1}{u_{\mathbf{k}}} e^{-\left(v_{\mathbf{k}} / u_{\mathbf{k}}\right) a_{\mathbf{k}}^{\dagger} a_{-\mathbf{k}}^{\dagger}}|0\rangle
$$

where

$$
u_{\mathbf{k}}=\frac{1}{\sqrt{2}} \sqrt{\frac{\tilde{\epsilon_{\mathbf{k}}}}{E_{\mathbf{k}}}+1} \quad v_{\mathbf{k}}=\frac{1}{\sqrt{2}} \sqrt{\frac{\epsilon_{\mathbf{k}}}{E_{\mathbf{k}}}-1}
$$

with $\tilde{\epsilon_{\mathbf{k}}}=\epsilon_{\mathbf{k}}+\frac{2 M_{0}}{N} U$, where $M_{0}$ is the number of particles in the condensate, and $\epsilon_{\mathbf{k}}=-2 t\left(\cos k_{x} a+\cos k_{y} a+\right.$ $\left.\cos k_{z} a\right)+6 t$ is the dispersion in the lattice which goes like $t a^{2} k^{2}=\hbar^{2} k^{2} / 2 m$ in the long wavelength limit. In order to perform our calculations we will introduce a cut-off defined by the point at which the kinetic energy becomes comparable to the on-site repulsion, i.e. $k_{c}=\frac{\sqrt{3} \pi}{a} \sqrt{\frac{U M_{0}}{t N}}$.

In the Bogolyubov state, "conservation" of bosons imposes the sum rule

$$
\begin{aligned}
M & =\sum_{\mathbf{k} \neq 0}\left\langle a_{\mathbf{k}}^{\dagger} a_{\mathbf{k}}\right\rangle+M_{0}=\sum_{\mathbf{k} \neq 0} v_{\mathbf{k}}^{2}+M_{0} \\
1 & =\frac{3}{16} a^{3} k_{c}^{3} \frac{N}{M}+M_{0} / M \\
1 & =\frac{3}{16} \pi^{3}\left(\frac{3 M_{0} U}{N t}\right)^{3 / 2} \frac{N}{M}+\frac{M_{0}}{M}
\end{aligned}
$$

which determines the number of particles in the condensate. In the Bogolyubov state we have a superfluid order parameter which corresponds to boson pair correlations

$$
\begin{aligned}
\left\langle a_{\mathbf{k}} a_{-\mathbf{k}}\right\rangle & =\frac{-v_{\mathbf{k}}}{u_{\mathbf{k}}} \\
& =-\sqrt{\frac{\left(\tilde{\epsilon}_{\mathbf{k}} / E_{\mathbf{k}}\right)-1}{\left.\tilde{\epsilon}_{\mathbf{k}} / E_{\mathbf{k}}\right)+1}} \\
& =-\sqrt{\frac{\epsilon_{\mathbf{k}}+\frac{2 M_{0} U}{N}-E_{\mathbf{k}}}{\epsilon_{\mathbf{k}}+\frac{2 M_{0} U}{N}+E_{\mathbf{k}}}}
\end{aligned}
$$

with $E_{\mathbf{k}}=\sqrt{\epsilon_{\mathbf{k}}} \sqrt{\epsilon_{\mathbf{k}}+4 \frac{M_{0}}{N} U}$ the quasiparticle excitation spectrum of the superfluid. Since at long-wavelengths it is a sound spectrum, the system superflows by the Landau argument ${ }^{14}$. Even though a Bose condensate is necessary for the Bose system to be a quantum liquid, the Bogolyubov pairs arising from the repulsion between atoms give the system the necessary rigidity to be superfluid. Therefore the superfluid order parameter is the amplitude of Bogolyubov pairs as whenever this amplitude vanishes, the system dissipates.
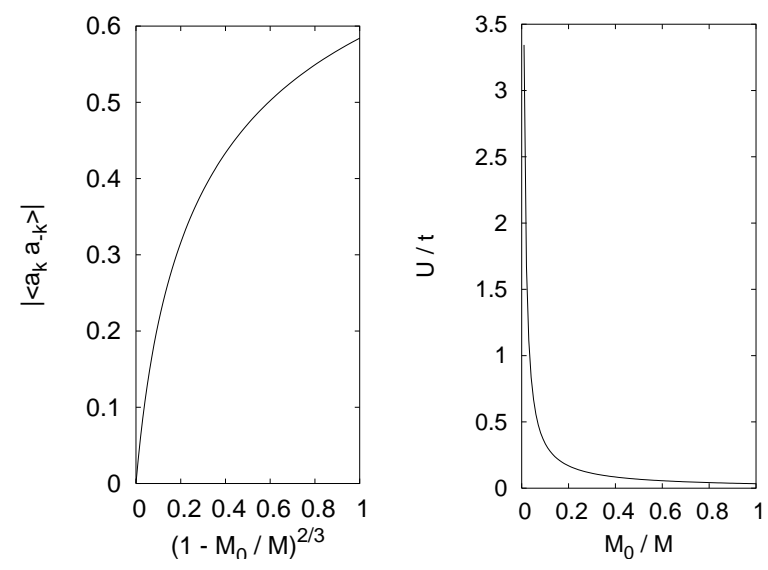

FIG. 2: (a) Dependence of the order parameter on the fraction of bosons in the condensate, (b) $U / t$ vs. $M_{0} / M$

In BEC's in an optical lattice the Mott transition is induced by making the wells on the lattice sites deeper $(\varepsilon$ is the lattice depth) thus suppressing the tunelling amplitude $t$ between near neighbor sites, i.e. making the bosons "heavy". Thus the relevant ratio of on-site repulsion $U$ to hopping $t$ increases. As this ratio increases, the superfluid order parameter increases as shown in the figure 2. The increase of the order parameter means that the superfluid phase is becoming more stable over the Bose condensed gas. On the other hand, we also plot the number of particles in the condensate. This number is becoming small as $U / t$ increases. As the condensate gets depleted the system is becoming "less fluid" and will solidify into a Mott insulating phase with a well defined number of bosons per site. Since the Bogolyubov order parameter will be zero in the quantum solid phase, there will be a discontinuous jump in the superfluid response at the Mott transition.

The Mott-insulating ground state wavefunction for $p N \leq M<(p+1) N$, with $p$, an integer is

$$
\left|\psi_{1}\right\rangle=\prod_{j=1}^{M-p N} a_{i_{j}}^{\dagger} \prod_{l=1}^{N}\left(a_{l}^{\dagger}\right)^{p}|0\rangle
$$

where $a_{i_{j}}$ and $a_{l}^{\dagger}$ are boson creation operators at lattice sites $i_{j}$ and $l$ respectively. For an incommensurate system, $p N<M<(p+1) N$, the ground state will not be 
strictly periodic as there are $M-p N$ that do not fill all lattice sites. No two of these extra bosons would occupy the same site when the system is in its ground state, but otherwise they will be randomly distributed. In real life, besides on-site interactions, there are interactions acting at long distances. They can be attractive or repulsive. These interactions are weaker than the on site interaction $U$, hence not important for a commensurate system. But if there is incommensuration, these interactions will probably play a role in determining the positions of the extra bosons.

Let us take a look at the expectation value of the superfluid order parameter in the Mott phase:

$$
\left\langle a_{\mathbf{k}} a_{-\mathbf{k}}\right\rangle=\frac{1}{N} \sum_{<i j\rangle} e^{i \mathbf{k} \cdot\left(\mathbf{r}_{i}-\mathbf{r}_{j}\right)}\left\langle\psi_{1}\left|a_{i} a_{j}\right| \psi_{1}\right\rangle
$$

where

$$
\left\langle 0\left|\prod_{l} a_{l}^{q_{l}} a_{i} a_{j} \prod_{l}\left(a_{l}^{\dagger}\right)^{q_{l}}\right| 0\right\rangle=\prod_{l \neq i, j}\left\langle 0\left|F_{i j} a_{l}^{q_{l}}\left(a_{l}^{\dagger}\right)^{q_{l}}\right| 0\right\rangle
$$

In the expression above, $q_{l}$ indicates the number of bosons in site $l$ and $F_{i j}$ is a function of bosonic operators on sites $i$ and $j$.

$$
\begin{aligned}
\prod_{l \neq i, j}\left\langle 0\left|F_{i j} a_{l}^{q_{l}}\left(a_{l}^{\dagger}\right)^{q_{l}}\right| 0\right\rangle & =\prod_{l \neq i, j}\left\langle 0\left|F_{i j} a_{l}^{q_{l}-1} a_{l}\left(a_{l}^{\dagger}\right)^{q_{l}}\right| 0\right\rangle \\
& =\prod_{l \neq i, j}\left\langle 0\left|F_{i j} a_{l}^{q_{l}-1} q_{l}\left(a_{l}^{\dagger}\right)^{q_{l}-1}\right| 0\right\rangle \\
& +\prod_{l \neq i, j}\left\langle 0\left|F_{i j} a_{l}^{q_{l}-1}\left(a_{l}^{\dagger}\right)^{q_{l}} a_{l}\right| 0\right\rangle \\
& =\prod_{l \neq i, j}\left\langle 0\left|F_{i j} a_{l}^{q_{l}-1} q_{l}\left(a_{l}^{\dagger}\right)^{q_{l}-1}\right| 0\right\rangle
\end{aligned}
$$

Continuing the process we find

$$
\begin{array}{r}
\prod_{l \neq i, j}\left\langle 0\left|F_{i j} a_{l}^{q_{l}-1} q_{l}\left(a_{l}^{\dagger}\right)^{q_{l}-1}\right| 0\right\rangle=\prod_{l \neq i, j} q_{l} !\left\langle 0\left|F_{i j}\right| 0\right\rangle \\
=\prod_{l \neq i, j} q_{l} !\left\langle 0\left|a_{i}^{q_{i}} a_{j}^{q_{j}} a_{i} a_{j}\left(a_{i}^{\dagger}\right)^{q_{i}}\left(a_{j}^{\dagger}\right)^{q_{j}}\right| 0\right\rangle
\end{array}
$$

Since there is a non matching number of operators acting on the ground state this yields 0 . The Bogolyubov order parameter is 0 in the Mott phase

$$
\left\langle a_{\mathbf{k}} a_{-\mathbf{k}}\right\rangle=0
$$

Actually this is obvious because the Mott state has a well defined number of bosons per site and number phase uncertainty makes the off-diagonal order zero. We thus see that, as anticipated, the only way to go into the Mott insulating phase is to have a discontinuous change in the superfluid order parameter. The Mott transition is discontinuous, i.e. first order, with the superfluid order parameter, and thus the superfluid response, experiencing a sudden jump to 0 .

The transition into the Mott insulating phase will occur when the Mott wavefunction (6) has less variational energy than the Bogolyubov superfluid wavefunction (2) With the Hamiltonian given by (1), the energy of the Mott state is found to be

$$
\begin{aligned}
&\left\langle\psi_{1}|\mathcal{H}| \psi_{1}\right\rangle=-t \sum_{i, \delta}\left\langle\psi_{1}\left|a_{i+\delta}^{\dagger} a_{i}\right| \psi_{1}\right\rangle \\
&-(\varepsilon+U) \sum_{i}\left\langle\psi_{1}\left|n_{i}\right| \psi_{1}\right\rangle+U \sum_{i}\left\langle\psi_{1}\left|n_{i}^{2}\right| \psi_{1}\right\rangle
\end{aligned}
$$

The first term corresponds to the kinetic energy. It destroys a boson in site $i$ and creates it in its nearest neighbor site $i+\delta$. Thus the lack of overlap of the resulting wavefunction with the ground state gives 0 . We get

$$
\begin{aligned}
&\left\langle\psi_{1}|\mathcal{H}| \psi_{1}\right\rangle=-(\varepsilon+U) M+U(M-p N)(p+1)^{2} \\
&+U[N-(M-p N)] p^{2} \\
&\left\langle\psi_{1}|\mathcal{H}| \psi_{1}\right\rangle=-\varepsilon M+2 p U M-(p+1) p N U
\end{aligned}
$$

We see that $2 p U M \geq(p+1) p N U$. After plenty of tedious algebra, with our cut-off method the variational energy the superfluid ground state can be estimated to be

$$
\begin{aligned}
\left\langle\psi_{0}|\mathcal{H}| \psi_{0}\right\rangle \simeq-\varepsilon M & +\frac{3}{32} \pi^{5}\left(\frac{3 M_{0} U}{t N}\right)^{5 / 2} N t \\
& +U N\left(\frac{3 M_{0} U}{N t}\right)^{3}\left(\frac{1}{27}+\frac{\pi^{3}}{8 \sqrt{3}}+\frac{27 \pi^{6}}{16^{2}}\right) \\
& -6 t M
\end{aligned}
$$

For $U / t$ small, we see that the interaction terms in the superfluid are almost irrelevant when compared with those of the Mott phase so the superfluid state is the stable one. We also notice that for not too large an $M$ the interaction terms in the superfluid grow a lot faster than the interaction terms in the Mott insulating phase. Hence for some critical $U / t$ the Mott insulating phase will become stable causing the system to undergo a quantum phase transition. Whether $M$ is commensurate or incommensurate with the lattice is irrelevant to the energetics: The state with all bosons localized is energetically favorable to the state with all bosons superfluid. Whether the instability of the superfluid is toward a phase with all bosons localized, or into a state with coexistence of a commensurate Mott insulator with the leftover bosons superfluid 
is not straightforward to answer variationally. It can be answered experimentally as in the case of coexistence the superfluid response will have a discontinuous jump to a smaller nonzero value, while in the case of all bosons localized the superfluid response will have a discontinuous jump to 0 .

The phase diagram of the simple Bose system at zero temperature shown in figure 1 differs considerably from the one proposed in the pioneering theoretical work on Bose systems ${ }^{2}$ and thus requires some comments. The first and, perhaps, most important difference is that we do not consider a phase only model as it is not appropriate to the Mott insulator at low densities. In that original work, the phase of the superfluid was disordered by the increasing repulsion thus leading to a transition. In such a transition the solid would have a supefluid order parameter within each well, but the phase of the order parameter will have become randomized exactly analogous to what happens in Josephson junction arrays when the charging energy is sufficiently large. Such an insulating phase does not correspond to the Mott insulator we studied here and cannot exist at small number of bosons per site as one needs a macroscopic number of particles to have a superfluid order parameter. Finally, we stress the the phase only model studied in the early work ${ }^{2.16}$ is a correct model of an array of Josephson coupled superfluid systems and should work for bosons in an optical lattice when the number of bosons per site is large enough to make superfluid order within each well possible. Josephson coupled systems can easily be studied in an optical lattice 19,20. It will be extremely interesting to see what the experimental phase diagram turns out to be.

The correct physics for the Bose system phase diagram can be easily differentiated experimentally as the original work would predict that the superfluid response goes continuously to zero at the transition. We predict that the superfluid response will have a sharp jump to zero at the transition. We predict a Mott transition irrespective of commensurability as long as the number of bosons per site is less than some critical density $n_{c}$. At high densities there should be a "crossover" into the physics of coupled Josephson arrays 2.16 .

Acknowledgements: We thank Ari Tuchman and Mark Kasevich for numerous and stimulating discussions, and for the extreme generosity with their data prior to publication. Bob Laughlin and Sandy Fetter provided very useful criticism and suggestions. Zaira Nazario was supported by The School of Humanities and Sciences at Stanford University. David I. Santiago was supported by NASA grant NAS 8-39225 to Gravity Probe B.
1 M. Greiner et. al., Nature 415, 39 (2002).

2 M.P.A. Fisher et. al., Phys. Rev. B. 40, 546 (1989).

3 K. B. Davis et. al., Phys. Rev. Lett. 75, 3969 (1995).

4 C. C. Bradley et. al., Phys. Rev. Lett. 75, 1687 (1995).

${ }_{5}^{5}$ M. H. Anderson et. al., Science 269, 198 (1995).

${ }^{6}$ M. R. Andrews et. al., Phys. Rev. Lett. 79, 553 (1997).

7 W. Ketterle et. al., Phys. Rev. Lett. 83, 2876 (1999).

8 A. Görlitz et. al., Phys. Rev. A 63, 041601 (2001).

${ }^{9}$ B. Damski et. al., Phys. Rev. Lett. 91, 080403 (2003).

10 G. G. Batrouni et. al., Phys. Rev. Lett. 89, 117203 (2002).

11 N. N. Bogolyubov, J. Phys. USSR 11, 23 (1947).

12 A. M. Ray et. al., J. Phys. B 36, 825 (2003).

13 Van Oosten et. al., Phys. Rev. A 63053601 (2001).
14 L. D. Landau, J. Phys. USSR 5, 71 (1941).

15 Ari Tuchman and Mark Kasevich, private communication of not yet published data that has been presented at several conferences. Preliminary data indicates the possibility that the transition is indeed first order under specific conditions.

16 Batrouni et. al., Phys. Rev. Lett. 65, 1765 (1990).

17 L. D. Landau and E. M. Lifshitz, Statistical Physics, (C)Reed Educational Publishing Ltd 1980.

18 P. W. Anderson, Rev. Mod. Phys. 38, 298 (1966).

19 B. P. Anderson, M. A. Kasevich, Science 282, 1686 (1999).

${ }^{20}$ F. S. Cataliotti et al., Science 293, 843 (2001). 